\title{
Development of a Multicenter Interventional Cardiology Database: The Blue Cross Blue Shield of Michigan Cardiovascular Consortium (BMC2) Experience
}

\author{
EVA KLINE-ROGERS, M.S., R.N.,* DAVID SHARE, M.D., M.P.H.,† DIANE BONDIE, B.S.,* \\ BRUCE ROGERS, B.S., * DEAN KARAVITE, B.S., *SHERRI KANTEN, R.N., PATRICIA WREN, R.N.,§ \\ CINDY BODURKA, R.N.,\|CATHY FISK, R.N.,\# JOHN MCGINNITY, M.S., P.A.-C., ** SUSAN WRIGHT, \\ R.N., $\dagger$ SUSAN FOX, R.N., $\ddagger$ KIM A. EAGLE, M.D.,* and MAURO MOSCUCCI, M.D.* ON BEHALF OF BLUE \\ Cross Blue Shield of Michigan Cardiovascular Consortium (BMC2)
}

From the *University of Michigan, Division of Cardiology, Blue Cross Blue Shield of Michigan Cardiovascular Consortium Coordinating Center, Ann Arbor; †Blue Cross Blue Shield Center for Health Care Quality, Detroit; $¥$ Spectrum Health, Grand Rapids; §St. Joseph Mercy Hospital, Ann Arbor; "Northern Michigan Hospital, Petoskey; "McLaren Regional Medical Center, Flint; ${ }^{* * H a r p e r ~ H o s p i t a l, ~ D e t r o i t ; ~}{ }^{+t}$ St. Joseph Hospital, Pontiac; and ${ }^{\ddagger \ddagger}$ Henry Ford Hospital, Detroit, Michigan

\begin{abstract}
The technical challenges in the development of a quality-controlled registry of percutaneous coronary interventions (PCIs) are currently unknown. This article describes the authors' experience in the development of a regional, quality-controlled PCI registry. In 1996, 16 centers in Michigan were invited to participate in a multicenter PCI registry. Nine centers agreed to a pilot data collection and, as of July 2001 , eight centers are still actively collecting data. An Oracle database was developed by the coordinating center. A common data collection form and a standard set of definitions were agreed on during several meetings. Data validity was insured through review of each form by a trained nurse, by automatic database diagnostic routines, and by site visits that included a review of the catheterization laboratory logs and a review of randomly selected charts. The average number of forms requiring query resolution was 33\% in 1997 (range 7-76\%), and it decreased to 5\% in 1999 (range 1.4-10\%). The most commonly queried variables were outcomes prior to discharge, lesion category, lesion complexity, date of birth, device used, gender, postprocedural percent stenosis, presence of left main disease, and MI date. Invalid dates, identification of the doctor, the presence of duplicate forms, and of duplicate outcomes were additional common queries generated by the internal diagnostic routines. In conclusion, the number of queries and diagnostic reports generated in the database suggests that the development of a quality-controlled PCI registry requires the institution of a careful diagnostic and data quality assessment system. (J Interven Cardiol 2002;15:387-392)
\end{abstract}

\section{Introduction}

The development of multicenter registries to measure outcomes is becoming increasing popular as health care costs continue to rise and medical practi-

Supported in part by a grant from the Blue Cross Blue Shield of Michigan Foundation, Detroit, Michigan.

Address for reprints: Mauro Moscucci, M.D., University of Michigan Medical Center, Taubman 3910, 1500 East Medical Center Dr., Ann Arbor, MI 48109-0022. Fax: (313) 936-5626; e-mail moscucci@umich.edu tioners are being closely scrutinized regarding appropriateness of procedures, resource use, and cost-effectiveness of care delivered. Health care purchasers and payors and governmental health care organizations, like the Health Care Financing Administration (HCFA), are requiring documentation of outcomes to validate that care was delivered effectively and appropriately.

As a subspecialty of cardiology, interventional cardiology continues to advance since 1977 when the first percutaneous revascularization was performed. ${ }^{1}$ With rapid evolution of technology, continued demand, and 
expanding indications for percutaneous procedures comes the need to measure outcomes in communitybased hospitals and tertiary care centers to insure that the high costs associated with these procedures are associated with optimal outcomes.

Outcome measurements have also been used as catalysts for quality improvement efforts. Some of the goals of quality improvement efforts are to insure appropriateness of procedures, cost-effectiveness, efficient delivery of care, and optimal resource use. One component of the quality improvement process is benchmarking, the process of identifying the best practice. By benchmarking, individuals and institutions are able to evaluate their own practices using the best practice as the criterion standard. Using this standard, individuals and institutions can plan and implement strategies to improve practice and outcomes. In the field of percutaneous coronary intervention (PCI), which is rapidly evolving, few well-established benchmarks are available. As a result, multicenter registries offer the opportunity to identify the best practices in real time on a regional basis, as they evolve.

The Blue Cross Blue Shield of Michigan Cardiovascular Consortium (BMC2) is a voluntary consortium of physicians, nurses, and administrators from eight hospitals in the state of Michigan who have agreed to participate in a multicenter registry of percutaneous coronary interventions (PCIs). The consortium is designed to extend over a 5-year period and incorporates a quality improvement phase. The purpose of the consortium is to develop a continuous quality improvement program, to prospectively evaluate appropriateness of percutaneous procedures, to develop and validate risk adjustment models for fatal and nonfatal outcomes of PCIs, and to apply these models in assessing the quality of care. This article describes the structure of data collection, validation procedures, and reporting of outcomes.

\section{Methods}

Participating Hospitals. In the summer of 1996, 16 academic and community hospitals in the Blue Cross Blue Shield of Michigan Centers of Excellence were asked to voluntarily participate in the registry. Nine hospitals agreed (three academic and six community) and were represented at the start-up meeting in early 1997. A 1-month pilot study was conducted in July 1997 followed by initiation of an ongoing registry on December 1, 1997.
Data Form. A one page (two-sided) data form that included demographic and procedural variables, past medical history, primary indication for PCI, priority, appropriateness, therapies, cardiac anatomy and function, and outcome data was developed from the input of physician representatives from the original nine hospitals. The data form went through several iterations before a final "draft" was implemented for the pilot study (Table 1). A list of standard definitions using the American College of Cardiology Guidelines as a reference was developed and sent to the sites with the data form. Definition of additional data elements not included in the American College of Cardiology database were agreed on by the participating centers. The data form was originally designed as a scannable form using Teleform technology (Cardiff Software Inc., Vista, CA, USA). More recently, dual manual data entry is used for transferring data into the database.

Data Collection. Participating sites submitted the protocol to their respective Institutional Review Boards for approval prior to initiation. Because the project is a registry for quality assessment and quality improvement with no experimental interventions, and given that no direct patient identifiers are included in the database, informed consent was waived by the institutions. Each site has a physician investigator and nurse coordinator for the study. The coordinators are required to keep an ongoing log of consecutive interventional patients with instructions to complete one data form per procedure. (If one patient has two sepa-

Table 1. BMC2 Dataform: Four Iterations

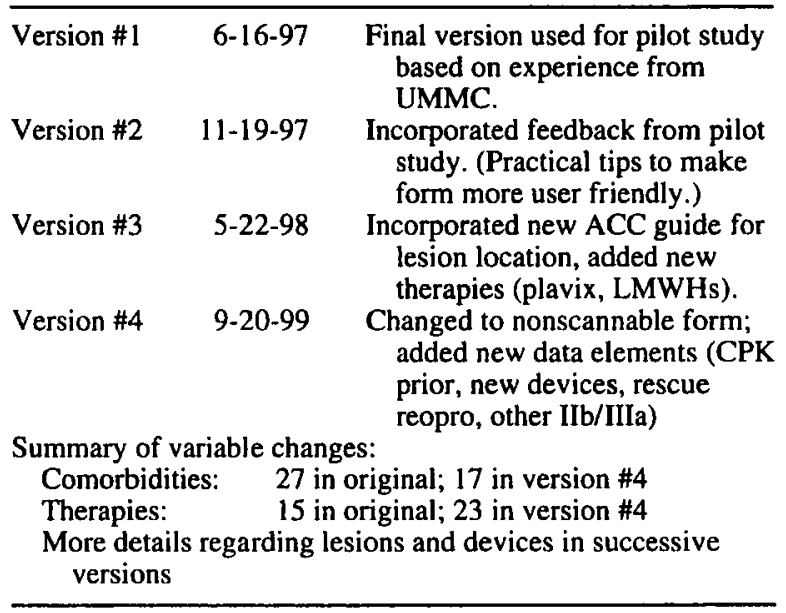

$\mathrm{ACC}=$ American College of Cardiology $\mathrm{BMC2}=$ Blue Cross Blue Shield of Michigan Cardiovascular Consortium; CPK = creatine phosphokinase; LMWH = low molecular weight heparin; UMMC = University of Michigan Medical Center. 
rate interventions during the same hospitalization, two separate data forms are completed.) Each data form has a unique identifier (form number) that is linked to patient identifiers at each site for purposes of query resolution. The study coordinators have 2 months after the end of each quarter to complete data forms on consecutive patients for that quarter. They send the data forms to the coordinating center where queries are resolved before data are imported and quarterly reports are generated.

Data Validation. All data goes through a three-step validation process before any reports are generated. The three steps include manual review for completeness and face validity, review of rejected data forms during the import process, and review of forms that fail diagnostic inquiries (Table 2).

When data forms arrive at the coordinating center, they are first checked for completeness by the coordinating center staff. If a variable is omitted, a query is sent to the appropriate coordinator via e-mail asking for the missing variable. No data forms are scanned into the staging tables awaiting import into the database until all fields are complete.

In addition, a random sample of data forms is checked for face validity. (All data forms from new sites and new coordinators are checked.) An example of review for face validity is checking for clinical consistency. For example, if nephropathy requiring dialysis is listed as an outcome, then an elevated creatinine level postprocedure would be expected. Similarly, if an intervention was performed within 24 hours of an acute myocardial infarction (MI), an elevated creatinine phosphokinase level should be recorded in the labs section and Q-wave MI should not be marked in the outcomes section unless that particular patient developed recurrent symptoms of an acute MI during the same hospitalization.

Until June 2000, after resolution of the data form omissions, the data forms were scanned using Teleform Software. Any questionable fields were highlighted and reviewed before acceptance. More re-

Table 2. BMC2 Data Process

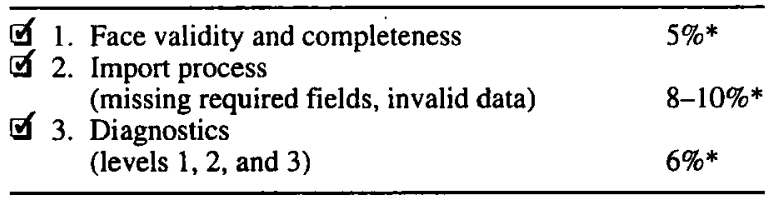

*Percent of data forms queried. BMC2 = Blue Cross Blue Shield of Michigan Cardiovascular Consortium. cently, the Teleform phase has been eliminated and dual manual data entry is used. Dual manual data entry is overall more reliable and, in the end, less time consuming. The data are then imported into staging tables awaiting import into the Oracle database. Approximately $8-10 \%$ of all data forms are rejected at this point due to preprogrammed constraints. Data forms will be rejected if required fields are missing or data are invalid (e.g., creatinine is recorded as 90 ).

After resolution of these queries, the data are imported into an Oracle database where a series of diagnostic reports are run to identify potential errors (Table 3). All Level 1 and Level 2 diagnostics are resolved before quarterly reports are printed.

Site Audits. All sites are audited twice yearly by a nurse practitioner from the coordinating center. (The coordinating center is audited by another BMC2 nurse coordinator.) During the site visit, a computerized printout of cases from each site is compared to the catheterization laboratory log to insure that all consecutive interventional procedures have been captured. If cases are missing, the study coordinator is required to complete the data forms and send them to the coordi-

Table 3. $\mathrm{BMC}^{2}$ Diagnostic Reports

Once the data are imported onto Oracle, a series of diagnostic
reports are run to identify potential errors. All Level 1 and
Level 2 diagnostics are resolved before quarterly reports are
printed.

Level 1

Invalid M.D. identifications

Duplicate forms

By procedure data and date of birth

By case number

Age exceptions $(<30$ or $>90)$

Procedure date not within study period

Procedure before admit date

Procedure date before date of myocardial infarction (MI)

Procedure date after discharge date

\section{Level 2}

Duplicate outcomes

Low ejection fractions $(<10 \%)$

Admit date not within study

Discharge date not within study

MI date not within study

Hospital stay $>90$ days

Hospital stay $<1$ day

Procedure $>30$ days after MI

Level 3

Duplicate diabetic entries (IDDM vs NIDDM)

Weight exceptions $(>300$ or $<100)$

Weight missing

MI present but no MI date

Check for invalid MI dates 
nating center after the site visit. A random $2 \%$ sampling of cases is audited for accuracy. The variables on the original data form are compared to the hospital chart. In addition, all hard outcomes (any coronary artery bypass grafting [CABG], death) are audited for accuracy.

\section{Results}

Participating Hospitals. Within the first 6 months, three of the nine original hospitals discontinued participation because they lacked resources to complete the data forms on consecutive interventional procedures. By the next calendar year, two of the original hospitals rejoined the registry resulting in a core group of eight hospitals (Fig. 1), three academic and five community. These eight hospitals represent a diverse patient population served by community and academic hospitals in the state of Michigan.

Data Form Evolution. Since the beginning of the registry, the BMC2 data form has gone through a total of four iterations. The second iteration incorporated feedback from the pilot study that included practical tips from the study coordinators to make the form more user friendly. Subsequent iterations have reflected changing technology in interventional cardiology like the addition of new therapies and devices (Table 1). The data form also includes a "flex box" where short-term variables for ad hoc analysis may be added or deleted. More recently, the data entry process was changed from scannable forms to dual manual data entry to ensure further accuracy in the data entry

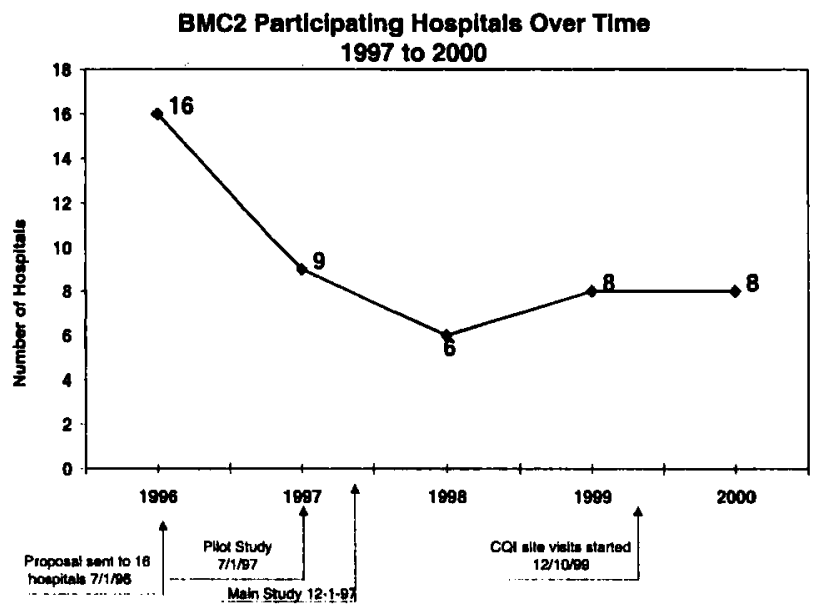

Figure 1. Number of participating hospital over time and study phases. and because the performance of the optical scanning system fell short of expectations.

Data Validity. Approximately 5\% of all data forms have at least one missing variable. The ten most frequently omitted variables are listed in Table 4 . Baseline demographics like age and gender, and outcomes are important variables that are occasionally missing from the submitted data form. An additional $10 \%$ of forms are rejected at the import phase, and $6 \%$ are currently rejected at the database level (Table 2). As shown in Figure 2 the overall number of queries has decreased by $30 \%$ since the pilot study. In the BMC2 experience with centers that were added at a later stage, the number of omitted variables (and all queries) decreases as the coordinator becomes more experienced, representing a definite learning curve. As a result of the data validity process, there is currently a $100 \%$ completeness for mandatory fields such as age, gender, or outcomes prior to discharge.

Missing cases are the most common problems identified during the site visits. The number of missing cases ranges from $1 \%$ to $18 \%$ of overall cases. Importantly, the missing cases are usually emergency procedures performed during weekends or after hours, and are more likely to be associated with adverse outcomes.

Quarterly Reports. Once the data are clean and complete, quarterly reports are printed for each site. These reports include a column that lists the collaborative data, a column for site data, and a third column with individual physician data. All sites and physicians are coded by number and only the individual site study coordinator has physician and patient identifiers. This allows individual practitioners and sites to compare their practices/outcomes with the collaborative. The quarterly reports include nine separate pages detailing demographics, case mix, preprocedure therapy, procedure data by patient, procedure data by lesion, outcomes in the laboratory, outcomes prior to discharge, and mortality. The mortality data are shown as observed and predicted. ${ }^{2,3}$

Table 4. Ten Most Frequently Queried Variables

\begin{tabular}{ll}
\hline Category & Post procedure stenosis \\
ACC lesion type & Gender \\
Number grafts $>70 \%$ & MI date \\
Device & LM disease \\
DOB & Outcomes prior to D/C \\
\hline
\end{tabular}

$\mathrm{ACC}=$ American College of Cardiology $\mathrm{D} / \mathrm{C}=$ discharge; $\mathrm{DOB}=$ date of birth; $\mathrm{LM}=$ left main; $\mathrm{MI}=$ myocardial infarction. 
\% Data Forms Requiring Query Resolution (Number of sites $=8$ )

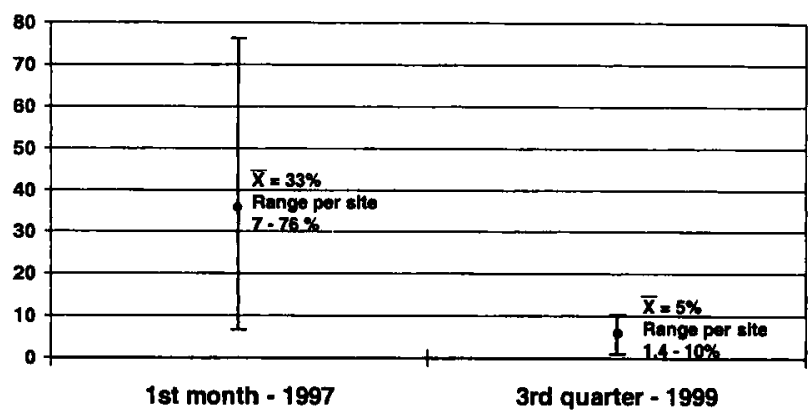

Figure 2. Average percentage and range of forms requiring query resolution at the beginning of the study and more recently.

\section{Discussion}

The BMC2 is one of several multicenter interventional registries within the United States designed to document procedural data and clinical outcomes in patients undergoing PCIs. The National Heart, Lung, and Blood Institute Percutaneous Transluminal Coronary Angioplasty (PTCA) Registries provided information on consecutive cases during two distinct time periods (1979 through 1981 and 1985 through 1986) ${ }^{4}$ from 16 participating North American hospitals, and has provided extensive data on outcomes of conventional percutaneous balloon angioplasty. More recently, the New Approaches to Coronary Intervention (NACI) Registry was developed to collect in-depth data about patients being treated with new interventional techniques 5 with an emphasis on stringent procedural data collection. Other examples of large registries at national and state levels include the National Cardiovascular Network Database (NCN), the American College of Cardiology National Data registry, the State of California Hospital discharge abstract data, and the state of New York Coronary Angioplasty Reporting System. So far, the State of California registry and the New York State registries have reported outcomes from data collected in 1989 and 1991 (New York). ${ }^{6,7}$ The NCN is a multicenter outcome-based organization established to foster interventional contracts with health care purchasers and to document clinical data and stimulate quality improvement. ${ }^{8}$ On a regional level, The Northern New England Cardiovascular Disease Study Group (NNECVDSG) is an ongoing voluntary consortium that has been collecting data on percutaneous revascularization procedures since $1990 .^{9}$
$\mathrm{BMC} 2$ is a consortium similar to the NNECVDSG, and it represents an example of a spontaneous collaborative effort at a regional level. Thus, since the initial introduction of PTCA in 1977, several registries have been collecting data on PCIs on a regional or national level to better understand clinical outcomes.

The data from the present registry show that outcome assessment does not end with the creation of a single center or a multicenter registry, but requires the institution of a process aimed toward the assurance of the quality of the data entered in the database, which is necessary to assure analytic rigor and credibility. Such assurance includes ascertainment of submission of consecutive cases, and of data completeness, particularly in relation to key fields like baseline demographics and major postprocedural outcomes. In addition, clinical inconsistencies and out of range data are other common problems in the data collection process that can be easily resolved with automatic internal queries and reports. Database efforts that fail to undertake this level of data scrutiny will be significantly weakened by essential data that are missing or inaccurate.

The process instituted in the present system includes review of charts of procedures where a major adverse outcome was reported, and an additional $2 \%$ random chart review. Obviously, review of every chart might further assure data quality. However, this effort would have insurmountable budgetary constraints for a voluntary consortium with limited funding like BMC2. However, no chart review at all could open the possibility of inaccurate or incomplete data collection, and of gaming. Thus, the authors believe that chart review limited to procedures where a major adverse outcome was reported and to an additional $2 \%$ of charts randomly selected is a reasonable compromise that can help in assuring the accuracy of the data collection process and in limiting gaming. They have found that these audits identify patterns of inaccuracy at a given institution that can be rectified through dialogue with the study coordinator. In addition, the comparison of the database logs with the procedures logs in the participating institutions appears to be the only way to assure the inclusion of consecutive cases.

Finally, the authors believe that the success of the BMC2 data collection effort reflects not only the process described here, but also the collaborative and voluntary nature of the consortium, the development of a common data form with a standard set of definitions, and the presence of dedicated clinical coordinators at each participating site. 


\section{Conclusion}

The BMC2 was designed to provide interventional cardiology practitioners with timely, valid information regarding current interventional practices and outcomes along with benchmarking data to use for quality improvement purposes. Analysis of the data so far collected and a quality improvement effort are currently underway.

\section{Appendix}

BMC2 Hospitals and Working Group Members. University of Michigan Health System in Ann Arbor (BMC2 Coordinating Center): Kim A. Eagle, M.D., F.A.C.C., Mauro Moscucci, M.D., Eva Kline-Rogers, M.S., R.N., Diane Bondie, B.S., Bruce Rogers, B.S., Jeanna Cooper, M.S.; Blue Cross Blue Shield of Michigan: David Share, M.D.; Northern Michigan Hospitals in Petoskey: William Meengs, M.D., Robert Sloan, M.B.A., Cindy Bodurka, R.N.; Spectrum Health Downtown in Grand Rapids: William McNamara, M.D., Ann M. Eward, Ph.D., Sherri Kanten, R.N., Renee Stamper, J.D.; McLaren Regional Medical Center in Flint: Anthony DeFranco, M.D., F.A.C.C., James Chambers, D.O., Cathy Fisk, R.N., Leisa Pertler, R.N., Stacey Somsky, R.N.; St. Joseph Mercy Hospital in Ann Arbor: Michael O'Donnell, M.D., Patricia Wren, R.N.; St. Joseph Mercy Hospital in Pontiac: Kirit Patel, M.D., Susan Wright, R.N., Bethany Smith, R.N.; Harper Hospital in Detroit: John McGinnity, M.S., P.A.-C., Cathy June, R.N.; Henry Ford Hospital in
Detroit: Vivian Clark, M.D., Phillip Kraft, M.D., Laurel Dvorak, R.N., Kelly Ryan, R.N., Margaret Fox, R.N., Cathy Lucarelli, R.N.

\section{References}

1. Gruentzig AR, Senning A, Siegenlhaler WE. Non-operative dilatation of coronary artery stenosis: Percutaneous transluminal coronary angioplasty. N Engl J Med 1979;301:61-68.

2. O'Connor GT, Malenka DF, Quinton $\mathrm{H}$, et al. Multivariate prediction of in-hospital mortality after percutaneous coronary interventions in 1994-1996. Northem New England Cardiovascular Disease Study Group. J Am Coll Cardiol 1999;34: 681-691.

3. Moscucci M, O'Connor GT, Ellis SG, et al. Validation of risk adjustment models for in-hospital percutaneous transluminal coronary angioplasty mortality on an independent data set. J Am Coll Cardiol 1999;34:692-697.

4. Detre K, Holubhov R, Kelsey S, et al. Percutaneous transluminal coronary angioplasty in 1985-1986 and 1977-1981: The NHLBI Registry. N Engl J Med 1988;318:265-270.

5. Steenkiste AR, Baim DS, Sipperly ME, et al. For the NACI Investigators: The NACI Registry: An instrument for the evaluation of new approaches to coronary intervention. Cathet Cardiovasc Diagn 1991;23:270-281.

6. Ritchie JL, Phillips KA, Luft HS. Coronary angioplasty statewide experience in California. Circulation 1993;88:27352743.

7. Hannan EL, Arani DT, Johnson LW, et al. Percutaneous transluminal coronary angioplasty in New York state: Risk factors and outcomes. JAMA 1992;268:3092-3097.

8. Peterson ED, Lansky AJ, Anstrom KJ, et al. Evolving trends in interventional device use and outcomes: Results from the $\mathrm{Na}$ tional Cardiovascular Network Database. Am Heart J 2000;139:198-207.

9. McGrath PD, Malenka DJ, Wennberg DE, et al. Changing outcomes in percutaneous coronary interventions: A study of 34,752 procedures in northern New England, 1990 to 1997. Northern New England Cardiovascular Disease Study Group. J Am Coll Cardiol 1999;34:674-680. 